\title{
Cinacalcet normalizza la calcemia sierica nei pazienti con iperparatiroidismo primitivo con controindicazioni alla chirurgia: studio randomizzato a doppio cieco
}

\author{
Marco Centanni ${ }^{1}$. Ilenia Gatto ${ }^{1}$
}

Pubblicato online: 14 luglio 2015

(C) Springer International Publishing AG 2015

Commento a:

Cinacalcet normalizes serum calcium in a double-blind randomized, placebo-controlled study in patients with primary hyperparathyroidism with contraindications to surgery.

A. Khan, J. Bilezikian, H. Bone, A. Gurevich, P. Lakatos, W. Misiorowski, L. Rozhinskaya, M.L. Trotman, M. Tóth.

Eur J Endocrinol (2015) 172(5):527-535

Il cinacalcet è un calciomimetico che modula allostericamente il recettore di membrana del calcio (CaSR), localizzato soprattutto a livello paratiroideo e renale, aumentandone la sensibilità. Attraverso questo meccanismo determina una riduzione dei livelli del PTH e della calcemia sierica. Il cinacalcet è attualmente approvato per la riduzione dell'ipercalcemia nei pazienti con: carcinoma paratiroideo, iperparatiroidismo primitivo (IPP) grave non idonei alla paratiroidectomia, iperparatiroidismo secondario con insufficienza renale in stadio terminale in terapia dialitica. In particolare, questo principio attivo è un'opzione terapeutica di prima scelta nei pazienti che non rientrino nei criteri di eleggibilità e/o che non risultino idonei al trattamento chirurgico, che lo rifiutino o che presentino una recidiva o una persistenza di malattia. Precedenti studi hanno dimostrato l'efficacia del cinacalcet nell'iperparatiroidismo primitivo ma il lavoro di Khan e collaboratori riporta i risultati del primo studio clinico controllato, randomizzato a doppio-cieco di fase 3, multicentrico e internazionale, sul trattamento con cinacalcet nei

M. Centanni

marco.centanni@uniroma1.it

1 Dipartimento di Scienze e Biotecnologie Medico-Chirurgiche, UOC di Endocrinologia, "Sapienza" Università di Roma, Latina, Italia pazienti con controindicazioni al trattamento chirurgico di paratiroidectomia.

Lo studio è stato condotto su 67 pazienti selezionati ed equamente distribuiti per caratteristiche antropometriche e cliniche, randomizzati in 33 trattati con cinacalcet e 34 trattati con placebo. La durata totale di 52 settimane era suddivisa in due periodi: le prime 28 dedicate alla randomizzazione, alla calibrazione del dosaggio e alla valutazione dell'efficacia terapeutica; le successive 24 settimane dedicate al trattamento di tutti i pazienti con cinacalcet. I parametri laboratoristici valutati ogni 3-4 settimane durante la randomizzazione sono stati: calcemia corretta per l'albumina, PTH, fosforemia, fosfatasi alcalina e vitamina $\mathrm{D}_{3}$. Sono stati inoltre valutati la qualità della vita relativa alla salute $\mathrm{e}$ gli effetti collaterali riconducibili al farmaco. La dose iniziale di cinacalcet era di $30 \mathrm{mg} \times 2 /$ die, quella mediana somministrata durante lo studio è stata di $60,2 \mathrm{mg} /$ die. Gli obiettivi di valutazione dell'efficacia terapeutica consistevano nella normalizzazione della calcemia, e/o una soglia minima di riduzione $\geq 1 \mathrm{mg} / \mathrm{dl}$ rispetto al valore basale e nella riduzione del PTH. La normalizzazione della calcemia e la sua riduzione $\geq 1 \mathrm{mg} / \mathrm{dl}$ sono state ottenute in una percentuale significativamente maggiore nei pazienti trattati con cinacalcet rispetto a quelli trattati con placebo $(75,8 \%$ vs $0 \%, 84,8 \%$ vs $5,9 \%$ rispettivamente). Inoltre, il cinacalcet ha determinato una riduzione significativa anche dei livelli di PTH, senza comunque ottenere la normalizzazione in tutti i pazienti. Il trattamento era scevro dagli effetti collaterali, principalmente nausea e spasmi muscolari, eccedenti quelli riportati in entrambi i gruppi e, soprattutto, non è stata rilevata alcuna ipocalcemia. La rilevanza di questi dati risiede nella conferma dell'efficacia e della sicurezza del cinacalcet, in uno studio multicentrico controllato che ne garantisce la validità nell'ipercalcemia non trattabile chirurgicamente, consentendone la registrazione con questa nuova indicazione terapeutica. 